\title{
Numerical Analysis of Oxygen Vacancy Distribution in Semiconductor Gas Sensors in the Cooling Process Based on the Model of Gradient-Distributed Oxygen Vacancies
}

\author{
Jianqiao LIU*, Zhaoxia ZHAI, Guohua JIN, Liting WU, Fengjiao GAO, Wusong HONG \\ College of Information Science and Technology, Dalian Maritime University, Linghai Road 1, Ganjingzi District, Dalian \\ 116026, Liaoning, China P.R. \\ crossref http://dx.doi.org/10.5755/j01.ms.26.2.21571
}

Received 04 September 2018; accepted 27 November 2018

\begin{abstract}
The oxygen vacancies $\left(\mathrm{V}_{\mathrm{O}}\right)$ play an essential role in the gas-sensing mechanism of semiconductor devices. A diffusion equation is established to describe the $\mathrm{V}_{O}$ behaviors during a cooling process based on the model of gradient-distributed oxygen vacancies. Numerical solutions of the diffusion equation are found to illustrate the $\mathrm{V}_{O}$ distribution in grains. The gradient of $V_{O}$ distribution is of negative dependence on the cooling rate, which also influences the average $V_{O}$ density in the depletion layer. The migration of oxygen vacancies in cooling process could be interrupted by quenching and it is restarted by the re-annealing process. The $\mathrm{V}_{O}$ distributing process is illustrated by three stages from initial uniform distribution to final gradient profile via a transient stage. The influence of $V_{O}$ distribution on gas-sensing characteristics of semiconductor grains is discussed. Potential opportunities are found to control the gas sensor characteristics by a designed annealing process.

Keywords: semiconductor, gas sensor, oxygen vacancy, diffusion equation, numerical analysis.
\end{abstract}

\section{INTRODUCTION}

Semiconductors are the one of the most popular research topics in recent decades because of their importance in preventing people from danger [1-3]. A semiconductor gas sensor detects stimulant gases by changing its resistance, which decreases in reducing gases but increases in oxidizing gases. The gas detection is completed by the reaction between reducing gas and adsorbed oxygen, or the competitive adsorption between adsorbed oxygen and oxidizing gas [4,5]. The adsorbed oxygen species come from the aerial oxygen, which interacts with the oxygen vacancy $\left(\mathrm{V}_{\mathrm{O}}\right)$ sites on the grain surface, capturing free electrons in the grain and leaving a depletion layer near grain surface [6]. Stoichiometric semiconductors are always insulative but they have semiconductive nature after the ionization of $\mathrm{V}_{\mathrm{O}}$, which results from the release of oxygen atom from crystal lattice during sintering $[7,8]$. Therefore, the behaviors of oxygen vacancies are essential questions in the gas-sensing mechanism of semiconductors.

It has been proved that oxygen atoms may escape from the lattice at a certain temperature, leaving oxygen vacancies in the grain [9]. The formation of oxygen defects usually takes place during sintering, in which the oxygen atom escapes from the crystal lattice according to Eq. 1,

$$
\mathrm{O}_{\mathrm{O}}^{\times} \leftrightarrow \mathrm{V}_{\mathrm{O}}^{\times}+\frac{1}{2} \mathrm{O}_{2},
$$

where $\mathrm{O}_{\mathrm{O}}{ }^{\times}$represents the oxygen atom at $\mathrm{O}$ site and $\mathrm{V}_{\mathrm{O}}{ }^{\times}$is the symbol of the oxygen vacancy.

\footnotetext{
${ }^{*}$ Corresponding author. Tel.: +86 411 84729934; fax: +86 41184729934

E-mail address: jqliu@dlmu.edu.cn (J. Liu)
}

The model of gradient-distributed oxygen vacancies was proposed for the $\mathrm{SnO}_{2}$ thin film gas sensors [10-12]. As described in these previous works, $\mathrm{V}_{\mathrm{O}}$ are involved into the grain when it grows up during the sintering. The defects would migrate during thermal vibration if the temperature is above absolute zero $\mathrm{K}$. The migration includes $\mathrm{V}_{\mathrm{O}}$ behaviors of diffusion and exclusion. The density gradient may lead to diffusion of the defects provided that the temperature is above the absolute zero K, following the Fick's second law. In another aspect, the cooling process may provide an excluding tendency to the defects because the crystal lattice is stable if it is free of defects $[13,14]$. Thus, the $V_{O}$ diffusion equation in the cooling process may be established, as Eq. 2:

$$
\frac{\partial N_{V}(r, t)}{\partial t}=D_{V}(t) \frac{\partial^{2} N_{V}(r, t)}{\partial r^{2}}-P(t) N_{V}(r, t),
$$

where $N_{V}(r, t)$ represents the $\mathrm{V}_{\mathrm{O}}$ density at the place of $r$ and time of $t$ in an ideal spherical grain, in which a sphere coordinates is established. Considering the symmetry of the sphere coordinates, only one-dimensional model is considered, as shown in Fig. 1. The first term of right side of the diffusion equation indicates the diffusion effect, which is expressed by the Fick's second law. $D_{V}(t)$ is the temperature dependent diffusion coefficient and it is formulated as Eq. 3:

$$
D_{V}(t)=D_{0} \exp \left(-\frac{E_{D}}{k T}\right)=D_{0} \exp \left[-\frac{E_{D}}{k\left(T_{0}-\beta t\right)}\right],
$$

where $D_{0}$ is the pre-exponent constant and $E_{D}$ is activated energy of diffusion. $k$ is the Boltzmann constant. $T_{0}$ is the 
initial temperature when the cooling process starts. $\beta$ is the cooling rate and $t$ is the time elapsed in the cooling process.

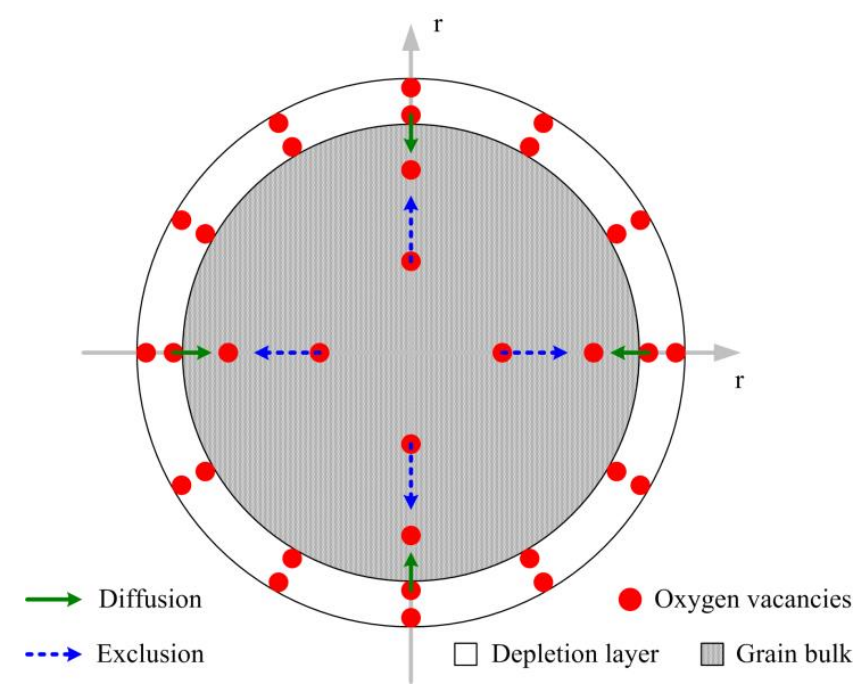

Fig. 1. Schematic drawing of the sphere coordinates in an ideal semiconductive grain with oxygen vacancies and their kinetics of diffusion and exclusion

The other term of right side of the diffusion equation denotes the exclusion effect. $P$ is the probability of the exclusion for a defect moving outwards to a nearby position in unit time and it can be formulated as Eq. 4:

$P(t)=v_{0} \exp \left(-\frac{E_{\varphi}-E_{0}}{k T}\right)=v_{0} \exp \left[-\frac{E_{\varphi}-E_{0}}{k\left(T_{0}-\beta t\right)}\right]$,

where $v_{0}$ is the thermal vibration frequency of the oxygen atom; $E \varphi$ represents the activated energy of defect migration; and $E_{0}$ is the unit energy decrease of the system that results from the one-step exclusion of defects. Therefore, the diffusion equation of Eq. 2 can be rewritten as Eq. 5, for which the analytical solution is not available.

$\frac{\partial N_{V}(r, t)}{\partial t}=\left\{\begin{array}{c}D_{0} \exp \left[-\frac{E_{D}}{k\left(T_{0}-\beta t\right)}\right] \frac{\partial^{2} N_{V}(r, t)}{\partial r^{2}} \\ -v_{0} \exp \left[-\frac{E_{\varphi}-E_{0}}{k\left(T_{0}-\beta t\right)}\right] N_{V}(r, t)\end{array}\right\}$.

In the present work, numerical methods are introduced in order to find the diffusion equation solutions, which may reveal the relationships among the parameters. The influences of cooling rate on the distribution of oxygen vacancies are discussed. The effect of annealing is revealed by comparing the defect distributions in quenched sample before and after annealing. The transient of the $\mathrm{V}_{O}$ distribution in the cooling process is also discussed.

\section{METHODS}

The computational tool of MATLAB R2014a (8.3.0.532) is used in order to find the numerical solution of the diffusion equation of Eq. 5. A discrete $N_{V}(r, t)$ is established in the array of $N_{V}(i, j)$, which comes from $N_{V}(r, t)=N_{V}(i \Delta r, j \Delta t)$, where $\Delta r$ and $\Delta t$ are the infinite small fragments in space and time. After two one-dimensional arrays of $D_{V}(j)$ and $P(j)$ are established, Eq. 6 can be obtained.

$$
N_{V}(i, j+1)=\left\{\begin{array}{c}
D_{V}(j) \frac{N_{V}(i+1, j)-2 N_{V}(i, j)+N_{V}(i-1, j)}{(\Delta r)^{2}} \Delta t \\
+[1-P(j) \Delta t] N_{V}(i, j)
\end{array}\right\} .
$$

Eq. 6 tells how the $\mathrm{V}_{\mathrm{O}}$ density at a point changes after a time of $\Delta t$ elapses under the effects of diffusion and exclusion from nearby positions. The initial condition that $N_{V}=0.5 N_{V S}$ is used with the presumption of uniform $V_{O}$ distribution throughout the grain. The boundary conditions contain two parts. The first one is the presumed constant $V_{O}$ density on grain surface $\left(N_{V S}\right)$. The second one is $\mathrm{d} N_{V}(r) / \mathrm{d} r=0$ at the center, meaning that the $\mathrm{V}_{\mathrm{O}}$ distribution is symmetrical in the grain. Thus, by using the discrete boundary conditions of Eq.7, the numerical solution is obtained in order to provide possibility to discuss the relationships among several fundamental parameters that determine the gas-sensing characteristics of semiconductor grains.

$$
\left\{\begin{array}{c}
N_{V}(0, j)=N_{V}(2, j) \\
N_{V}\left(\frac{R_{C}}{\Delta r}, j\right)=N_{V S}
\end{array},\right.
$$

where $N_{V S}$ is the $\mathrm{V}_{\mathrm{O}}$ density at the grain surface.

The obtained $\mathrm{V}_{\mathrm{O}}$ distribution in the grain could be used to calculate the electrical properties of semiconductor grains if the defects are assumed to be first-order ionized, according to Poisson's equation as Eq. 8:

$\frac{d^{2} V(x)}{d x^{2}}=\frac{q\left[N_{V}(x)\right]}{\varepsilon}, 0 \leq x \leq w$,

where $\varepsilon$ is the permittivity and $w$ is the depletion layer width. Then, potential for electrons $(V)$ is obtained by using the boundary conditions of $V(w)=0$ and $\mathrm{d} V(w) / \mathrm{d} x=0$. Thus, the reduced resistance $\left(R / R_{0}\right)$ is exponential to the potential barrier height at grain surface $\left(q V_{S}\right)$, which equals to $q V(0)$, as expressed in Eq. 9:

$\frac{R}{R_{0}}=\exp \left(\frac{q V_{S}}{k T}\right)$

where $R_{0}$ is the flat-band resistance and $T$ is the operating temperature. The sensor response $(S)$ to reducing gas is defined as the ratio of the resistance in air $\left(R_{a}\right)$ to the one in a reducing target gas $\left(R_{g}\right)$, as $S=R_{a} / R_{g}$. When the grain is exposed to reducing gases, the adsorbed oxygen on grain surface is consumed by reducing gas molecules. Parts of the electrons, which are used to be captured by the adsorbed oxygen, are released back to the depletion layer, lowered grain resistance. The parameter of $\alpha$ is used to indicate the proportion of the captured electrons that are released back to depletion layer after gas exposure. In the one-dimensional model, the depletion layer width after gas exposure could be expressed as $w_{g}=(1-\alpha) w$. Therefore, the sensor response could be calculated. In the following calculations, the parameters are set as follows:

$D_{0}=0.0431 \mathrm{~m}^{2} / \mathrm{s}[15]$,

$E_{D}=2.7 \mathrm{eV}[15]$

$v_{0}=10^{14} \mathrm{~s}^{-1}[16]$

$\varepsilon=10^{-10} \mathrm{~F} / \mathrm{m}[17]$,

$w=4 \mathrm{~nm}[8]$, 
$\alpha=0.5, R_{C}=25 \mathrm{~nm}, T_{0}=823 \mathrm{~K}$ and $E_{D^{-}} E \varphi+E_{0}=0.1 \mathrm{eV}$ [12].

\section{RESULTS}

The influence of cooling rate on the gradient distribution profile of oxygen vacancies in a $25 \mathrm{~nm}$ semiconductor grain is shown in Fig. 2.

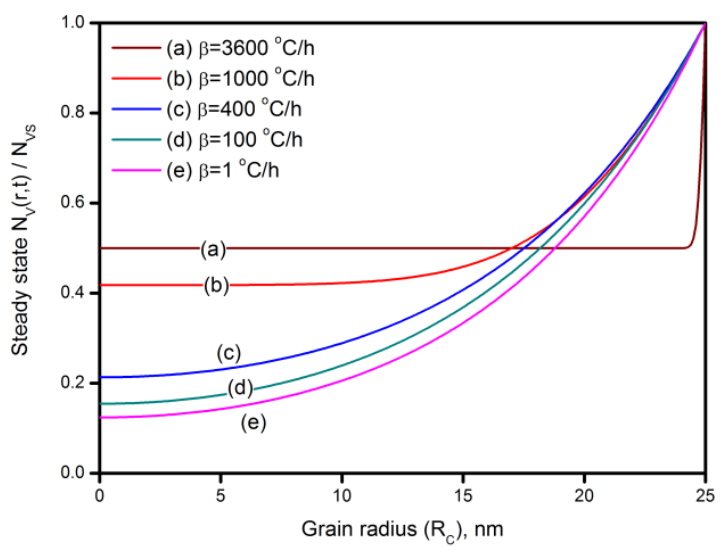

Fig. 2. Influence of cooling rate on the gradient distribution profile of oxygen vacancies in semiconductor grain with radius of $25 \mathrm{~nm}$

The $\mathrm{V}_{\mathrm{O}}$ has various distribution profiles that determined by the cooling rate. For the quenched sample with the largest cooling rate of $3600{ }^{\circ} \mathrm{C} / \mathrm{h}$, the initial $\mathrm{V}_{\mathrm{O}}$ does not have sufficient time to migrate. So they are frozen at the place where they are at the starting of the cooling process, leaving a nearly uniform distribution throughout the grain except the region near the surface. For the slowly-cooled sample, the initial $\mathrm{V}_{\mathrm{O}}$ migrates under the effect of diffusion and exclusion, forming a gradient distribution in the grain. The gradient is dependent on the cooling rate, which has a negative relationship with each other.

Fig. 3 shows the influence of cooling rate on the steady state $V_{O}$ density at the center of grain, where the defect density is the smallest throughout the grain.

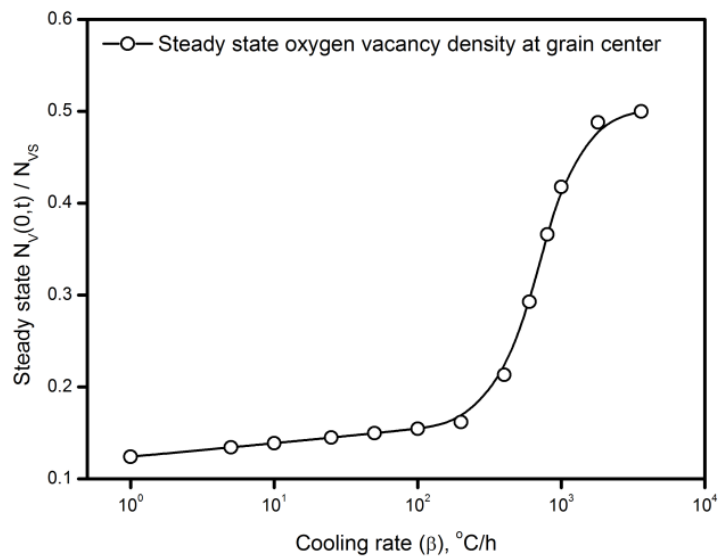

Fig. 3. The relationship between cooling rate and steady state oxygen vacancy density at the center of grain

It controls the density difference between the surface and center. Within the cooling rate concerned, there are two regions where the vacancy density has the linear correlation with the cooling rate. One is $\beta=1-200{ }^{\circ} \mathrm{C} / \mathrm{h}$ and the other is $\beta=400-1000{ }^{\circ} \mathrm{C} / \mathrm{h}$. It infers that the $\mathrm{V}_{\mathrm{O}}$ gradient can be easily controlled by the cooling rate in the two regions mentioned above.

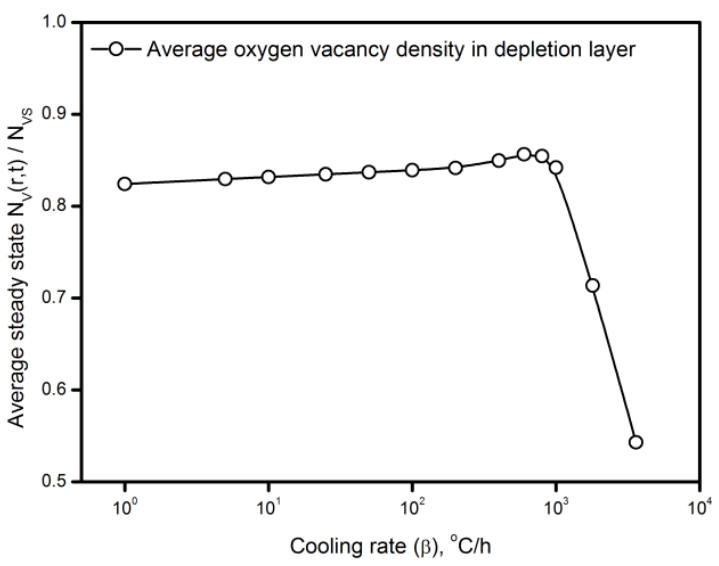

Fig. 4. The influence of cooling rate on the average oxygen vacancy density in the depletion layer of semiconductor grain

The gas-sensing characteristics of semiconductor grain are determined by the ionized donors, acted by the oxygen vacancies, in the depletion layer. The influence of cooling rate on the average $\mathrm{V}_{O}$ density in the depletion layer is revealed in Fig. 4. For the quenched sample, the average $V_{O}$ density is rather low so that it performed a little response to the stimulant gas [12]. Along with the decrease of cooling rate, the $\mathrm{V}_{\mathrm{O}}$ amount in depletion layer dramatically increases until it reaches the peak at $\beta=600^{\circ} \mathrm{C} / \mathrm{h}$. Then, it has a slight decrease with the cooling rate. Considering the determination of $\mathrm{V}_{\mathrm{O}}$ amount in depletion layer on the sensor performances, it is possible to control the sensor properties by adjusting the cooling rate in the fabrication process, in which an optimized cooling rate may be found. A designed fabrication process may be completed by controlling the cooling rate and time for an expected $\mathrm{V}_{\mathrm{O}}$ distribution as well as gas-sensing characteristics of sensors.

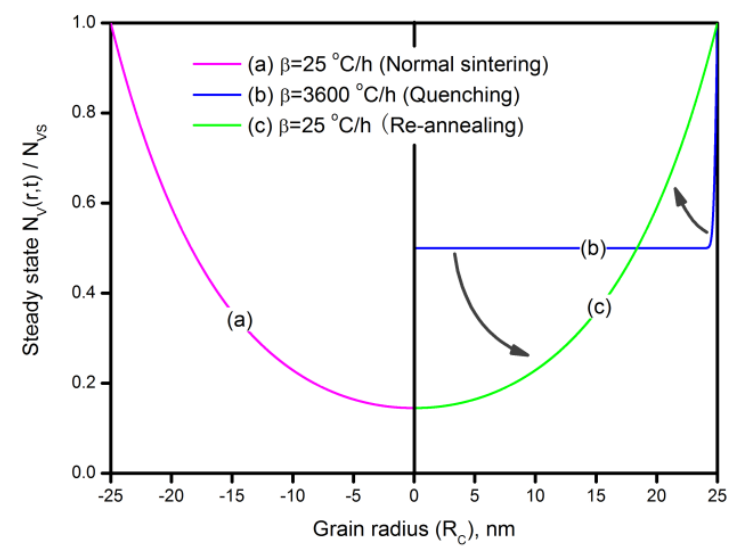

Fig. 5. The re-annealing effect of steady state oxygen vacancy distribution profile in a semiconductor grain, which is quenched with $\beta=3600^{\circ} \mathrm{C} / \mathrm{h}$ and then re-annealed with $\beta=25^{\circ} \mathrm{C} / \mathrm{h}$

The re-annealing effect is discussed based on the simulation results in Fig. 5. For a normal sintered grain, the $\mathrm{V}_{\mathrm{O}}$ distribution is shown as Fig. 5 a after a cooling rate of $25^{\circ} \mathrm{C} / \mathrm{h}$ is conducted. Meanwhile, the vacancy distribution for a quenched grain is shown as Fig. $5 \mathrm{~b}$ if the cooling rate of $3600{ }^{\circ} \mathrm{C} / \mathrm{h}$ is used. However, if a re-annealing process 
with cooling rate of $25^{\circ} \mathrm{C} / \mathrm{h}$ is employed to the quenched grain, the final vacancy distribution, Fig. $5 \mathrm{c}$, is found to be the same as the normal sintered one. It indicates that the fast cooling process interrupts the $\mathrm{V}_{\mathrm{O}}$ migration and the reannealing process restarts it again. This conclusion provides potential opportunities to adjust the properties of an existing semiconductor gas sensor by a designed annealing process. For instance, after a long-term usage with repeated heating, a gas sensor may fail to remain its consistency in sensor characteristics, which usually degraded to a low level. Based on the discussions above, the sensor could be recovered by using the re-annealing technique, which provides a re-distribution of oxygen vacancies and therefore adjusts the sensor properties.

The transient distribution of oxygen vacancies in a semiconductor grain during the cooling process is illustrated in Fig. 6 according to the numerical solution of Eq. 5, in which $R_{C}=25 \mathrm{~nm}$ and $\beta=100^{\circ} \mathrm{C} / \mathrm{h}$. The initial uniform $\mathrm{V}_{\mathrm{O}}$ distribution is driven to distribute in a gradient profile. It describes that the distribution of oxygen vacancies experiences three stages in the cooling process. At the starting stage, the $\mathrm{V}_{\mathrm{O}}$ density is uniform throughout the grain. Then, the $V_{O}$ migrates under the effects of diffusion and exclusion at the transient stage. Finally, the distribution reaches the steady state in a gradient profile.

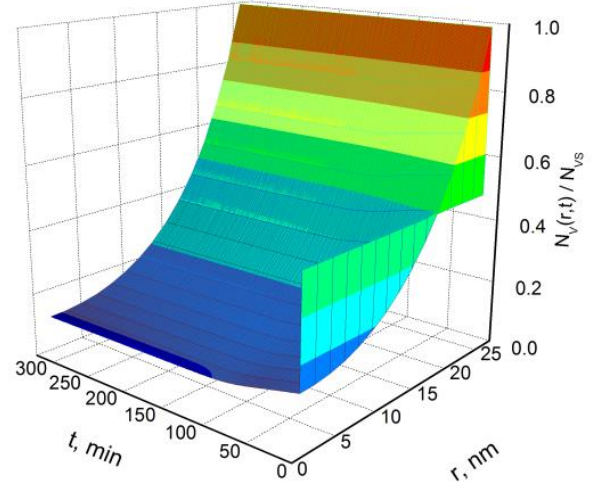

Fig. 6. Time-dependent oxygen vacancy distribution in a $25 \mathrm{~nm}$ semiconductor grain from initial uniform distribution to steady state gradient distribution

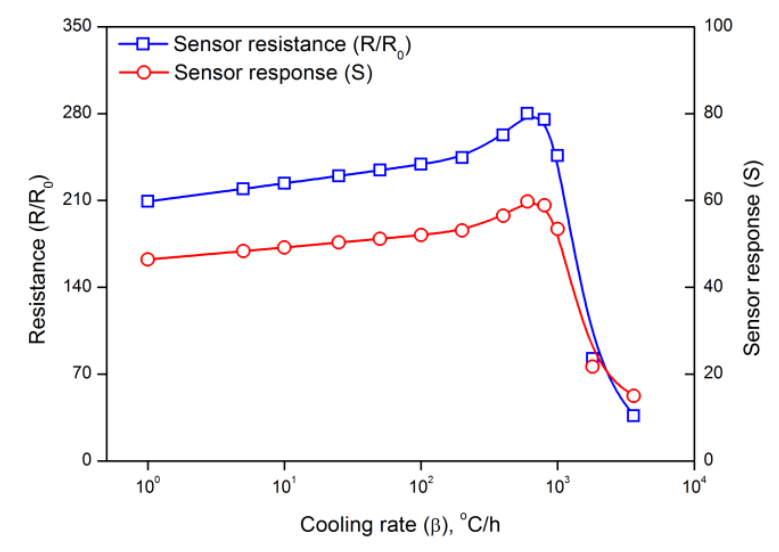

Fig. 7. Influences of cooling rate on the gas-sensing characteristics of resistance and response to reducing gas at the operating temperature of $25^{\circ} \mathrm{C}$

The calculated gas-sensing characteristics of the semiconductor grain with radius of $25 \mathrm{~nm}$ at the operating temperature of $25^{\circ} \mathrm{C}$ are shown in Fig. 7. It is observed that the reduced resistance $\left(R / R_{0}\right)$ and response to reducing gas $(S)$ increase with cooling rate before they reach the maximum at $\beta=600{ }^{\circ} \mathrm{C} / \mathrm{h}$. Then, both of the sensor properties appear dramatic decreases when fast cooling is conducted. The correlations are in agreement with the average $\mathrm{V}_{\mathrm{O}}$ density in the depletion layer. Thus, the sensor performances could be controlled by the $\mathrm{V}_{\mathrm{O}}$ distribution, which is determined by the cooling rate in the fabrication process.

\section{DISCUSSION}

The gas-sensing mechanism of semiconductors is divided into three levels, namely the receptor function, the transducer function and the utility factor [18]. The receptor function is the most essential one, which describes how a single grain respond to a stimulant gas. It includes several topics, such as the adsorbed oxygen, the depletion layer, the power law as well as the oxygen vacancy behavior. According to the results above, the behaviors of $\mathrm{V}_{\mathrm{O}}$ in the cooling process comprises formation and migration, which is completed under the effects of diffusion and exclusion. The $\mathrm{V}_{\mathrm{O}}$ distribution in the semiconductor grain is controlled by the cooling rate and determines the gas-sensing characteristics. The diffusion equation for $\mathrm{V}_{\mathrm{O}}$ migration is established and numerical solutions are found. The calculation results provide mathematical contributions to the receptor function of gas-sensing mechanism of semiconductors.

While focusing on the receptor function, the present work uses Schottky barrier model as transducer function to calculate the gas-sensing characteristics of semiconductor grains. However, an actual sensor body is an assembly of tiny semiconductor grains, meaning that the utility factor is needed in the evaluation of sensor performances. Further researches could be made by incorporating the gas-diffusion theory $[19,20]$ into the present model for a comprehensive simulation of actual sensor properties.

In the calculation, there are several presumptions, which need to be discussed. First, the present onedimensional model is different from the $3 \mathrm{D}$ grains in actual sensors. In the modeling, an actual grain is assumed to be an ideal sphere. Considering the symmetry of the sphere model, the one-dimensional model is used for simplification in calculation, which may bring derivation to the results. Fortunately, the effects of grain shape have been formulated. The expressions in one-dimensional model could be modified by incorporating shape factors [21, 22]. Therefore, the present model has the potential to be applied in actual three-dimensional grains.

Furthermore, the total amount of $\mathrm{V}_{\mathrm{O}}$ in the grain should be a dynamic parameter during the cooling process, which provides an excluding tendency to the defects. However, the exclusion may result in $\mathrm{V}_{\mathrm{O}}$ gradient in distribution and lead to diffusion from surface to center. Hence, the decrease in $\mathrm{V}_{\mathrm{O}}$ total amount is countered due to the diffusion. It is noted that the $\mathrm{V}_{\mathrm{O}}$ in the grain bulk and on the grain surface may change with each other under the thermodynamics. Also, the $\mathrm{V}_{\mathrm{O}}$ on the surface may interact with aerial atmosphere and change the value of $N_{V S}$. However, there is few studies on surface $V_{O}$, which has not been mathematically described 
yet. Thus, a constant $N_{V S}$ value is used as one of the boundary conditions to find numerical solutions. Further studies in quantitative manners are expected on the surface defects.

It is reserved that all the oxygen vacancies are assumed to be first-order ionized in the calculation of gas-sensing characteristics. The degree of ionization may influence several factors, such as migration of $\mathrm{V}_{\mathrm{O}}$, interaction between defects and potential barrier at grain boundaries. The migration of $\mathrm{V}_{\mathrm{O}}$ would make impacts on the diffusion coefficient $\left(D_{V}\right)$ and exclusion probability $(P)$. However, both of them are evaluated without considering the $\mathrm{V}_{\mathrm{O}}$ ionization degree because temperature is more decisive in the calculation of $D_{V}$ and $P$. Furthermore, the interaction between $\mathrm{V}_{\mathrm{O}}$ is neglected because the amount of $\mathrm{V}_{\mathrm{O}}$ is only a small percentage of the $\mathrm{Sn}$ and $\mathrm{O}$ amount in the lattice. In addition, the potential barrier at grain boundaries is calculated by the Poisson's equation of Eq. 8, in which the space charge is acted by the first-order ionized $V_{O}$ in the depletion layer.

\section{CONCLUSIONS}

In the present work, the $\mathrm{V}_{\mathrm{O}}$ migrations in the semiconductor grain are investigated and a diffusion equation is established to describe their behaviors during the cooling process. Numerical solutions of the diffusion equation are found to reveal the influence of cooling rate on the $\mathrm{V}_{\mathrm{O}}$ distribution. The fast cooling or quenching leads to an almost uniform $V_{O}$ distribution throughout the grain while the slow cooling benefits the establishment of a gradient distribution. The gradient is of negative dependence on the cooling rate. The average $V_{O}$ density in the depletion layer has a slight decrease with the cooling rate when $\beta<600^{\circ} \mathrm{C} / \mathrm{h}$. The re-annealing process of a quenched grain may restart the distributing process. This phenomenon provides potential opportunities to adjust the gas sensor properties by a designed annealing process. Three stages of $\mathrm{V}_{\mathrm{O}}$ distributing process in the cooling process are summarized. The gas-sensing characteristics of semiconductor grains could be controlled by the $\mathrm{V}_{O}$ distribution as well as the cooling rate in the fabrication process.

\section{Acknowledgments}

This work is financially supported by the National Natural Science Foundation of China (Grant No. 11704055), the Liaoning Natural Science Foundation (Grant No. 20180510021, the Joint Research Fund Liaoning-Shenyang National Laboratory for Materials Science), the Dalian High-level Talents Innovation Supporting Program (Grant No. 2017RQ073) and the Fundamental Research Funds for the Central Universities (Grant No. 3132019212, 3132019348).

\section{REFERENCES}

1. Song, Z., Wei, Z., Wang, B., Luo, Z., Xu, S., Zhang, W., Yu, H., Li, M., Huang, Z., Zang, J. Sensitive RoomTemperature $\mathrm{H}_{2} \mathrm{~S}$ Gas Sensors Employing $\mathrm{Sno}_{2}$ Quantum Wire/Reduced Graphene Oxide Nanocomposites Chemistry of Materials 28 (4) 2016: pp. 1205-1212. https://doi.org/10.1021/acs.chemmater.5b04850

2. Liu, H., Li, M., Voznyy, O., Hu, L., Fu, Q., Zhou, D., Xia, Z., Sargent, E.H., Tang, J. Physically Flexible, RapidResponse Gas Sensor Based on Colloidal Quantum Dot Solids Advanced Materials 26 (17) 2014: pp. $2718-2724$. https://doi.org/10.1002/adma.201304366

3. Rahimi, R., Raghavan, S., Shelton, N.P., Penigalapati, D., Balling, A., Woodworth, A.A., Denig, T., Stinespring, C.D., Korakakis, D. Investigation of NanoThin B-Sic Layers for Chemical Sensors MRS Proceedings 1056 2007: pp. $1056-\mathrm{HH} 1008-1005$. https://doi.org/10.1557/PROC-1056-HH08-05

4. Liu, J., Lu, Y., Cui, X., Geng, Y., Jin, G., Zhai, Z. GasSensing Properties and Sensitivity Promoting Mechanism of $\mathrm{Cu}$-Added $\mathrm{Sno}_{2}$ Thin Films Deposited by Ultrasonic Spray Pyrolysis Sensors and Actuators B: Chemical 248 2017: pp. $862-867$ https://doi.org/10.1016/j.snb.2017.01.057

5. Yamazoe, N., Shimanoe, K. Theory of Power Laws for Semiconductor Gas Sensors Sensors and Actuators B: Chemical 128 (2) 2008: pp. 566-573. https://doi.org/10.1016/j.snb.2007.07.036

6. Yamazoe, N., Fuchigami, J., Kishikawa, M., Seiyama, T. Interactions of Tin Oxide Surface with $\mathrm{O}_{2}, \mathrm{H}_{2} \mathrm{O}$ and $\mathrm{H}_{2}$ Surface Science 86 (1) 1979: pp. 335-344. https://doi.org/10.1016/0039-6028(79)90411-4

7. Morrison, S.R. Semiconductor Gas Sensors Sensors and Actuators 2 (44) 1982: pp. 329-341. https://doi.org/10.1016/0250-6874(81)80054-6

8. Liu, J., Liu, X., Zhai, Z., Jin, G., Jiang, Q., Zhao, Y., Luo, C., Quan, L. Evaluation of Depletion Layer Width and Gas-Sensing Properties of Antimony-Doped Tin Oxide Thin Film Sensors Sensors and Actuators B: Chemical 220 (15) 2015: pp. $1354-1360$. https://doi.org/10.1016/j.snb.2015.07.065

9. Morrison, S.R. Mechanism of Semiconductor Gas Sensor Operation Sensors and Actuators 11(3) 1987: pp. $283-287$. https://doi.org/10.1016/0250-6874(87)80007-0

10. Liu, J., Gao, Y., Wu, X., Jin, G., Zhai, Z., Liu, H. Inhomogeneous Oxygen Vacancy Distribution in Semiconductor Gas Sensors: Formation, Migration and Determination on Gas Sensing Characteristics Sensors 17 (8) 2017: pp. 1852. https://doi.org/10.3390/s17081852

11. Liu, J., Gong, S., Fu, Q., Wang, Y., Quan, L., Deng, Z., Chen, B., Zhou, D. Time-Dependent Oxygen Vacancy Distribution and Gas Sensing Characteristics of Tin Oxide Gas Sensitive Thin Films Sensors and Actuators B: Chemical 150 (1) 2010: pp. 330-338. https://doi.org/10.1016/j.snb.2010.06.065

12. Liu, J., Gong, S., Quan, L., Deng, Z., Liu, H., Zhou, D. Influences of Cooling Rate on Gas Sensitive Tin Oxide Thin Films and a Model of Gradient Distributed Oxygen Vacancies in $\mathrm{Sno}_{2}$ Crystallites Sensors and Actuators B: Chemical 145 (2) 2010: pp. 657-666. https://doi.org/10.1016/j.snb.2010.01.015

13. Shimizu, Y., Kobayashi, N., Uedono, A., Okada, Y. Improvement of Crystal Quality of Gainnas Films Grown by Atomic Hydrogen-Assisted Rf-Mbe Journal of Crystal Growth 278 (1-4) 2005: pp. $553-557$. https://doi.org/10.1016/j.jcrysgro.2004.12.114

14. Zhang, M., Lin, C., Weng, H., Scholz, R., Gösele, U. Defect Distribution and Evolution in $\mathrm{He}^{+}$Implanted $\mathrm{Si}$ 
Studied by Variable-Energy Positron Beam Thin Solid Films 333 (1-2) 1998: pp. 245-250.

https://doi.org/10.1016/S0040-6090(98)00808-6

15. Maier, J., Göpel, W. Investigations of the Bulk Defect Chemistry of Polycrystalline Tin (Iv) Oxide Journal of Solid State Chemistry 72 (2) 1988: pp. 293-302. https://doi.org/10.1016/0022-4596(88)90032-1

16. Kittel, C. Introduction to Solid State Physics, John Wiley \& Sons, Inc., 2004. https://doi.org/10.1063/1.3060399

17. Malagù, C., Guidi, V., Stefancich, M., Carotta, M.C., Martinelli, G. Model for Schottky Barrier and Surface States in Nanostructured N-Type Semiconductors Journal of Applied Physics 91 (2) 2002: pp. 808-814. https://doi.org/10.1063/1.1425434

18. Yamazoe, N., Shimanoe, K. New Perspectives of Gas Sensor Technology Sensors and Actuators B: Chemical 138 (1) 2009: pp. $100-107$. https://doi.org/10.1016/j.snb.2009.01.023
19. Sakai, G., Matsunaga, N., Shimanoe, K., Yamazoe, N. Theory of Gas-Diffusion Controlled Sensitivity for Thin Film Semiconductor Gas Sensor Sensors and Actuators B: Chemical 80 (2) 2001: pp. 125-131. https://doi.org/10.1016/s0925-4005(01)00890-5

20. Liu, J., Gong, S., Xia, J., Quan, L., Liu, H., Zhou, D. The Sensor Response of Tin Oxide Thin Films to Different Gas Concentration and the Modification of the Gas Diffusion Theory Sensors and Actuators B: Chemical 138 (1) 2009: pp. 289-295. https://doi.org/10.1016/j.snb.2009.02.018

21. Yamazoe, N., Shimanoe, K. Roles of Shape and Size of Component Crystals in Semiconductor Gas Sensors Ii. Response to $\mathrm{No}_{2}$ and $\mathrm{H}_{2}$ Journal of the Electrochemical Society 155 (4) 2008: pp. J93 - J98. https://doi.org/10.1149/1.2832655

22. Yamazoe, N., Shimanoe, K. Roles of Shape and Size of Component Crystals in Semiconductor Gas Sensors I. Response to Oxygen Journal of the Electrochemical Society 155 (4) 2008: pp. J85 - J92. https://doi.org/10.1149/1.2832655 